\title{
Formulation and Evaluation of Indomethacin Microspheres using natural and synthetic polymers as Controlled Release Dosage Forms
}

\author{
Chinna Gangadhar B. ${ }^{* 1}$, Shyam Sunder R. ${ }^{1}$, Vimal Kumar Varma. M. ${ }^{1}$, Sleeva Raju M. ${ }^{2}$, Sai Kiran M. ${ }^{3}$ \\ ${ }^{1}$ Department of Pharmaceuticals and Fine Chemicals, University College of Technology, Osmania \\ University, Hyderabad 500007, AP, India \\ ${ }^{2}$ Department of Microbiology, Don Bosco Academy, Nalgonda 508001, AP, India \\ ${ }^{3}$ Department of Pharmacology, Nalanda College of Pharmacy, Nalgonda 508001, AP, India
}

\begin{abstract}
Purpose: The aim of this study was to formulate and evaluate microspheres as controlled release preparations of a highly water-insoluble drug, Indomethacin, using natural polymer, Egg albumin; semi synthetic polymer, Ethyl cellulose and Synthetic polymer, Meth acrylic acid esters (Eudragit L 100) as the retardant materials.

Methods: Microspheres were prepared by solvent evaporation method using an acetone / liquid paraffin system and Phase separation co-acervation method using petroleum ether and coconut oil as dispersion and continuous phase systems. Magnesium stearate was used as the droplet stabilizer and n-hexane was added to harden the microspheres. The prepared microspheres were evaluated for their micromeritic properties, drug content and encapsulation efficiency and characterized by Fourier transform infrared spectroscopy (FT-IR), and scanning electron microscopy (SEM). The in vitro release studies was performed by buffer change method to mimic Gas Intestine Tract(GIT) environment in pH 1.2, carbonate buffer (acidic) and $\mathrm{pH} 7.4$, phosphate buffer (Alkaline).

Results: The prepared microspheres were pale yellow, free flowing and spherical in shape. The mean particle size of the microspheres was found in the range of 150 to $400 \mu \mathrm{m}$. The drug-loaded microspheres showed $70-86 \%$ of entrapment and release was extended up to 6 to $8 \mathrm{~h}$ releasing $86 \%$ of the total drug from the microspheres. The infrared spectra showed stable character of Indomethacin in the drug-loaded microspheres and revealed the absence of drug-polymer interactions. Scanning electron microscopy study revealed that the microspheres were spherical and porous in nature.

Conclusion: The best-fit release kinetics was achieved with Koresmeyer-Peppas plot followed by zero order and First order. The release of Indomethacin was influenced by the drug to polymer ratio and particle size \& was found to be both diffusion and dissolution controlled.
\end{abstract}

Keywords: Indomethacin, Egg albumin, Eudragit, Ethyl cellulose, FT-IR, SEM, Solvent Evaporation technique, Phase Separation technique, Microspheres.

\section{Introduction}

The efficacy of a drug in a specific application requires the maintenance of appropriate drug blood level concentration during a prolonged period of time. However the conventional administration of drugs, gives a poor control of the concentration of these substances in plasma because of variations in the concentration of the bioactive product, once a specific dose has been administered [1]. The conventional dosage systems can give rise to alternative periods of inefficacy or toxicity. These difficulties have been called for the development of new administration techniques for bioactive compounds, directed towards attaining the steady state plasma concentration [2]. In the recent years, considerable attention has been focused on the development of Novel Drug Delivery Systems (NDDS). The reason for this paradigm shift is due to the low development cost and time required for developing a NDDS for the existing drugs rather developing a new drug molecule [3-4]. In the form of NDDS, existing drug molecule can get a new life, thereby increasing the market value and product patent life. Controlled release, prolonged action, sustained release, extended release, depot dosage forms are terms used to identify these drug delivery systems that are designed to achieve prolonged therapeutic effect by continuously releasing medication over an extended period of time after administration of single dose [5]. Number of advances took place in the field of Controlled drug delivery systems in the last few decades. During the preliminary stages of research on controlled drug delivery, major accent was focused on the development of zero-order devices [6]. The primary objective of zero-order release is to up-hold constant drug concentration in blood for a prolonged period of time. Microspheres have played a vital role in the development of controlled/sustained release drug delivery systems [7-8]. Microspheres have been of particular interest from the pharmaceutical point of view providing the possibility to achieve sustained and controlled drug release. Controlled drug delivery using biodegradable polymeric carries has gained increasing interest in last two decades. In a majority of studies the homo and copolymer have been used for drug delivery application because they can be fabricated into a variety of morphologies, including films, rods, and micro particles by compression molding [9], solvent casting [10], solvent evaporation technique [11] and phase separation technique [12]. After the employment in the body, 
biodegradable polymers of natural and synthetic origin like Eudragit, ethyl cellulose and egg albumin have a unique advantage that after performing their function they degrade into non toxic monomers [13]. Administration of drugs in the form of microspheres, usually improves the treatment by providing the localization of the active substance at the site of action and by prolonging release of drug. Furthermore sensitive drug such as peptides and protein may be protected against chemical and enzymatic degradation when entrapped in microspheres. Wide ranges of microencapsulation techniques have been developed; the selection of the techniques depends on the nature of the polymer, the drug, and the intended use. When preparing controlled release microspheres, the choice of the optimal method has utmost importance for the efficient entrapment of the active substance [14]. Pharmaceutically acceptable techniques using hydrophobic biodegradable polymers as matrix materials include Emulsion-solvent evaporation, Phase separation (non solvent and solvent partitioning), Interfacial polymerization and Spray drying [15]. Indomethacin (IND) was selected as a mode drug which is a non-steroidal anti-inflammatory drug (NSAID) that reduces fever, pain, and inflammation. IND was selected mainly because of its side effects such as stomach irritation or intestinal bleeding or ulcers and can increase blood pressure and decrease kidney function, it is important to decrease used dosages and side effects during the treatment [16]. One of the possibilities to do it is direct delivery of the drug to the target area of the body by external magnetic field.

\section{Materials and methods}

Indomethacin (KAPL, Bangalore), Eudragit (Ranbaxy laboratories limited), Egg albumin (SD fine chemicals), Ethyl cellulose (SD fine chemicals), Heptane (Ranbaxy laboratories limited), Petroleum ether (Reachem laboratories limited), N-hexane (Finar Reagents), Magnesium stearate (SD fine-chemicals limited), Liquid paraffin (Qualigens Fine chemicals), Acetone (SD fine-chemicals limited) and all other solvents and reagents used were of analytical grade.

\section{Experiment}

Indomethacin microspheres were formulated using two techniques i.e. phase separation and solvent evaporation method depending upon the type of polymer employed for the formulation of microspheres. Biodegradable polymers employed were Eudragit (synthetic), ethyl cellulose (semi synthetic) and egg albumin (natural). In the formulations concentration of polymers were varied from $1-2-3 \% \mathrm{w} / \mathrm{v}$ respectively to study their effects on the drug release. The objective of varying the concentration of the polymers was to achieve optimized formulations, which would give a sustained release of the drug over a period of 8 hours.

\section{Preparation of Polymeric-Indomethacin microspheres}

Phase separation emulsion polymerization method was employed for the preparation of microspheres. Procedure in brief, polymeric drug solution was prepared by adding $1 \mathrm{~g}$ of drug (Indomethacin) to $10 \mathrm{ml}$ of $10 \%$ solution of egg albumin and stirred continuously until uniform dispersion. In a separate beaker, to $86 \mathrm{ml}$ of coconut oil $1 \mathrm{ml}$ of $0.5 \%$ sodium lauryl sulphate was added to give organic phase. Polymeric drug solution was added drop-wise using 22 gauge needle into the organic phase and stirred continuously to form uniform dispersion. The temperature of the solution was gradually increased to $80^{\circ} \mathrm{C}$ and stirred at this temperature for 2 hours. The solution was cooled to room temperature with continuous stirring using mechanical stirrer. After the room temperature was attained, $1 \mathrm{ml}$ of formaldehyde and $20 \mathrm{ml}$ of $\mathrm{n}$ Hexane was added to separate the microspheres from the organic phase which were filtered and separated. The obtained microspheres were washed thrice with $\mathrm{n}$-Hexane then followed by distilled water, dried and stored in air tight containers until further analysis. Solvent evaporation method was employed for the preparation of microspheres, procedure in brief, $1 \mathrm{gm}$ of Polymer was dissolved in $20 \mathrm{ml}$ of acetone and ethanol solution. To the above polymeric solution, $0.5 \mathrm{gm}$ of Indomethacin was added and dispersed. The resultant mixture was added drop-wise using 22 gauge needle into $100 \mathrm{ml}$ oil phase containing $50 \mathrm{ml}$ of coconut oil and $50 \mathrm{ml}$ of light liquid paraffin containing $0.2 \mathrm{ml}$ of Tween 80 . The resultant dispersion was stirred continuously using mechanical stirrer at $400 \mathrm{rpm}$ for 3-4 hours for ethyl cellulose and $1500 \mathrm{rpm}$ for 8 hours for Eudragit. It results in the formation of microspheres which were collected and washed with n-Hexane to remove the oil content followed by washing with distilled water thrice. The microspheres were then filtered, dried and stored in air tight container until further analysis. During the preparation procedure, emphasis is to be given on rpm and temperature for size and spherecity of the microspheres.

\section{Characterization}

The prepared microspheres were characterized by Fourier Transformed Infrared Spectroscopic analysis, The FT-IR spectral measurements were taken at ambient temperature using a Shimadzu, Model 8033 (USA) using $\mathrm{KBr}$ pellet method by applying $6000 \mathrm{~kg} / \mathrm{cm}^{2}$ pressure to study the polymer-drug interactions. The SEM analysis of the microspheres was carried out by using Jeol JSM 5300, Japan, to determine size, shape and 
surface morphology of the prepared microspheres.

\section{Evaluation \\ Content uniformity / drug loading}

The prepared microspheres were powdered and passed through sieve no (85/120). The powder retained on the sieve 120 was taken for content uniformity studies. A weight of powder containing $100 \mathrm{mg}$ of the drug was taken in a $100 \mathrm{ml}$ standard volumetric flask. To this of $0.1 \mathrm{~N} \mathrm{NaOH}$ solution was added and made upto the mark with $0.1 \mathrm{~N} \mathrm{NaOH}$ solution and kept overnight. The final solution was filtered using what man filter paper. From this $10 \mathrm{ml}$ was pipetted out into a $100 \mathrm{ml}$ standard volumetric flask and made upto the volume with $0.1 \mathrm{~N} \mathrm{NaOH}$ solution and estimated spectrophotometrically for drug content.

\section{Entrapment efficiency}

To evaluate the amount of the drug inside the microspheres, an indirect method was used. Aliquots from the filtered solutions remaining after removal of the microspheres were assayed spectrophotometrically. The amount of drug entrapped was calculated from the difference between the total amount of drug added and the amount of drug found in the filtered solution. About $100 \mathrm{mg}$ of microspheres were completely dissolved in $500 \mathrm{ml}$ of phosphate buffer solutions $(\mathrm{pH}$ 7.4), and stirred for $1 \mathrm{~h}$. Then, $2 \mathrm{ml}$ of solution was filtered and the concentration of drug was determined spectrophotometrically by UV. Efficiency of drug entrapment was calculated in terms of percentage drug entrapment (PDE) as per the following formula:

PDE $=($ Practical drug loading/Theoretical drug loading) $\times 100$

\section{In vitro drug release studies}

The In vitro release of drug from the microspheres was carried out in Paddle type dissolution tester-USP XXII, TDT-08L, with auto sampler containing $900 \mathrm{ml}$ of $\mathrm{pH} 1.2$ buffer for the first two hours followed by $\mathrm{pH} 7.4$ phosphate buffer for the next six hours, which was maintained at $37 \pm 0.5^{\circ} \mathrm{C}$ and at a stirring speed of $100 \mathrm{rpm}$. About $5 \mathrm{ml}$ of the Sample was withdrawn for every one hour and analyzed for drug content spectrophotometrically. The released data obtained were fitted into various mathematical models to know which mathematical model is best fitting the obtained release profile.

\section{Results and Discussion FT-IR analysis}

Indomethacin and one of the formulations of each polymer were subjected to FT-IR spectroscopic analysis, to ascertain whether there is any interaction between the drugs and the polymers used. The FT-IR spectra obtained is given in Fig. (1). The characteristic peaks of the pure drug were compared with peaks obtained from their respective formulations and are given in the tables and for Indomethacin and formulations respectively. From the data obtained it was observed that characteristic peaks appears with identical or with minor differences, at frequencies $424.35 \mathrm{~cm}^{-1}$ (C-N stretch), $451.36 \mathrm{~cm}^{-1}$ (C-O stretch of $\left.\mathrm{CH}_{3} \mathrm{O}\right), 464.86 \mathrm{~cm}^{-1}(\mathrm{C}=\mathrm{C}$ stretch of aromatic ring), 669.32 (C-Cl stretching), 1018.45 $\mathrm{cm}^{-1}$ (C-O stretch of primary alcohol) for Indomethacin and formulations, from the peaks it was evident that there was no chemical interaction between the drug and polymers.

\section{Scanning electron microscopy}

Scanning electron microscopy (SEM) is one of the most commonly used method for characterizing drug delivery systems, owing in large part of simplicity of sample preparation and ease of operation. Scanning electron microscopy was carried out in order to characterize surface morphology of the microspheres. In this study the morphological observations were carried out to study the surface morphology of microspheres. SEM micrographs and typical surface morphology of the microspheres are given in figures 2-4. It was observed that microspheres were spherical in nature [Fig. (2-4).], the microspheres ranged in size 150 to $408 \mu \mathrm{m}$.

\section{Content uniformity}

The test for content uniformity was carried out to ascertain whether the drug is uniformly distributed in the formulation. The results obtained are reported in Table. (1). From the results it can be inferred that there is proper distribution of Indomethacin in the microspheres and the deviation is within acceptable limits.

\section{Encapsulation efficiency}

The encapsulation efficiency was performed to find out the amount of drug that gets encapsulated in the microspheres so that sufficient amount of drug is present in the microspheres to ensure the drug remains in the therapeutic range once it enters the systemic circulation. The encapsulation efficiency was found to be higher in case of Egg albumin (86\%), when compared to the Eudragit (74\%) and Ethyl cellulose $(70 \%)$. Drug loading was further found to increase with the increase in the concentration of encapsulating polymer.

It is clear from Fig. (5). that, with the increase in the polymer concentration there was increase in the encapsulation efficiency of the drug which can be attributed to the increased availability of the polymer for encapsulating the drug i.e. Indomethacin. 


\section{In vitro release studies}

The in vitro release studies were carried out by buffer change method to mimic the GIT environment. It was carried out for all the formulations in both acidic $(\mathrm{pH} 1.2)$ media for the first two hours and basic ( $\mathrm{pH} \mathrm{7.4)} \mathrm{media} \mathrm{for} \mathrm{the}$ next 6 hours. The in vitro release profiles are given in Fig. (6-8).

Formulations with varying concentrations of polymer i.e. Eudragit, egg albumin and ethyl cellulose $(1: 1,1: 2$ and $1: 3)$ were used to study the effect of polymer concentration on the drug release from the microspheres.

For the initial 2 Hours i.e. in 1.2pH buffer the drug release was found to be low (less than 10\%) in all the cases, indicating that the microspheres did not release the drug in the acidic media, however when transferred into intestinal media there was slow dissolution of the polymer resulting in the slow release of the drug and it can also be attributed to the fact that the swelling of microspheres in the acidic media was low. However the basic $\mathrm{pH}$ of the media was able to penetrate the microsphere and dissolve the matrix to release the drug by diffusion.

In all the formulations, with the increase in the polymer concentration, the rate and amount of drug release was found to decrease, which can be attributed to the greater binding of the drug with the polymer. As the concentration of polymer increases, larger amounts of drug got binded in the polymer matrix as a result the rate of drug release from the microspheres decreases. The results obtained were fitted into various mathematical models to know which mathematical model is best fitting the release profile. The parameters, $\mathrm{n}$ and $\mathrm{k}$, were also determined for Koresmeyer-Peppas equation to know the release mechanism.

\section{Conclusion}

Microspheres as controlled release preparations were formulated and evaluated containing highly water-insoluble drug, Indomethacin, using natural polymer, Egg albumin; semi synthetic polymer, Ethyl cellulose and Synthetic polymer, Meth acrylic acid esters (Eudragit $L$ 100) as the retardant materials by complex coacervation method and emulsion solvent evaporation method respectively where the polymer concentration was varied keeping the drug concentration constant. The mean particle size of the microspheres was found in the range of 150 to $400 \mu \mathrm{m}$. The drug-loaded microspheres showed $70-86 \%$ of entrapment efficiency. The encapsulation efficiency was found to be higher in case of Egg albumin (86\%), when compared to the Eudragit (74\%) and Ethyl cellulose (70\%). Drug loading was further found to increase with the increase in the concentration of encapsulating polymer. The infrared spectra showed stable character of Indomethacin in the drug-loaded microspheres and revealed the absence of drug-polymer interactions. From SEM it was evident that the microspheres were spherical and porous in nature. In-vitro release was extended up to 6 to $8 \mathrm{~h}$ releasing $86 \%$ of the total drug from the microspheres. In all the formulations, with the increase in the polymer concentration, the rate and amount of drug release was found to decrease, which can be attributed to the greater binding of the drug with the polymer. From the mathematical models it was evident that the release of Indomethacin was influenced by the drug to polymer ratio and particle size \& was found to be both diffusion and dissolution controlled. This study illustrates a novel method for preparing microspheres to be used as controlled release dosage forms such that the drug is protected in the inner biocompatible polymeric matrix dictates the transport properties. These relatively porous and highly stable microspheres could also be applied in cell immobilization for biotechnology applications.

\section{Acknowledgment}

We take this opportunity to express our deep sense of gratitude and thanks to all the teaching and non-teaching staff of Dept. of Pharmaceuticals and Fine Chemicals, University College of Technology, Osmania University, Hyderabad, for their valuable guidance, personal assistance and motivation to carry out this work.

\section{References}

[1] Bhaskar Mazumder., Sanjib Bhattacharya., Bibhash Mohanta., Sanjay Dey., Anindya Maity. (2009) Int J PharmTech Research, 1(3), 905-913.

[2] Sunit Kumar Sahoo., Abdul Arif Mallick., Barik B.B., Prakash Ch Senapati. (2005) Tropical journal of Pharmaceutical Research, 4 (1), 369-375.

[3] Cheu S.J., Chen R.I., Chen P.F., Lin W.J. (2001) Journal of Microencapsulation, 18(5), 559-565.

[4] Chandy A., Sudish Rail., Manigauha A., Suresh Sahu. (2009) J. Pharma. Dosage Forms and Tech, 1(1), 29-32.

[5] Meral Yuce., Kandemir Canefe. (2008) Turk J. Pharm. Sci, 5 (3), 129-142.

[6] Perumal D., Dangor C.M., Alcock R. S., Hurbans N., Moopanar K.R. (1999) Journal of Microencapsulation, 16(4), 475-487.

[7] Deore B.V., Mahajan H.S., Deore U.V. (2004) Int J ChemTech Research, 1(3), 634-642.

[8] Tamer Guneri., Mesut Arici., Gokhan Ertan. (2004) Fabad j. pharm. Sci, 29, 177184.

[9] Kotadiya R., Patel V., Patel H., Koradiya H. (2009) Int J Green Pharmacy, 14, 58-62. 
[10] Waree Tiyaboonchai., Garnpimol C. Ritthidej. (2003) Songklanakarin J. Sci. Technol, 25(2), 245-254.

[11] Pachuau L., Sarkar S., Mazumder B. (2008) Tropical Journal of Pharmaceutical Research, 7 (2), 995-1002.

[12] Ibrahim M. El-Bagory., Ehab A. Hosny., Saleh A. Al-Suwayeh., Gamal M. Mahrous., Fahad I. Al-Jenoobi. (2007) Saudi pharm Journal, 15(3-4), 213-217.

[13] Radi Hejazi., Mansoor Amiji. (2002) Int $J$ Pharmaceutics, 235, 87-94.
[14] Sinjan De., Dennis Robinson. (2003) Journal of Controlled Release, 89, 101112.

[15] Suranjana Roy., Manjusree Pal., Bijan K. Gupta. (1992) Pharmaceutical research, 9(9), 1132-1136.

[16] Mostafa Saffari., Malihe Shahbazi., Mehdi Shafiee Ardestani. (2008) Nature Preceding, doi:10.1038/npre: 1544.1. 
Table 1- Content uniformity data for Indomethacin loaded microspheres.

\begin{tabular}{|l|l|c|c|c|c|}
\hline Sr. No & $\begin{array}{l}\text { Formulation of } \\
\text { Indomethacin }\end{array}$ & Trial1 & Trial 2 & Trial 3 & $\begin{array}{l}\text { Average } \\
\text { Mean(mg) }\end{array}$ \\
\hline 1 & Eudragit-F1 & 70.3 & 70.9 & 70.5 & 70.57 \\
\hline 2 & F2 & 72.4 & 72.6 & 72.5 & 72.5 \\
\hline 3 & F3 & 74.9 & 74.2 & 74.6 & 74.57 \\
\hline 4 & Egg albumin- F1 & 81.7 & 81.9 & 81.3 & 81.64 \\
\hline 5 & F2 & 83.5 & 83.2 & 83.9 & 83.53 \\
\hline 6 & F3 & 86.9 & 86.6 & 86.3 & 86.6 \\
\hline 7 & Ethyl cellulose- F1 & 65.1 & 65.6 & 65.5 & 65.4 \\
\hline 8 & F2 & 68.1 & 68.6 & 68.5 & 68.4 \\
\hline 9 & F3 & 70.5 & 70.1 & 70.4 & 70.33 \\
\hline
\end{tabular}
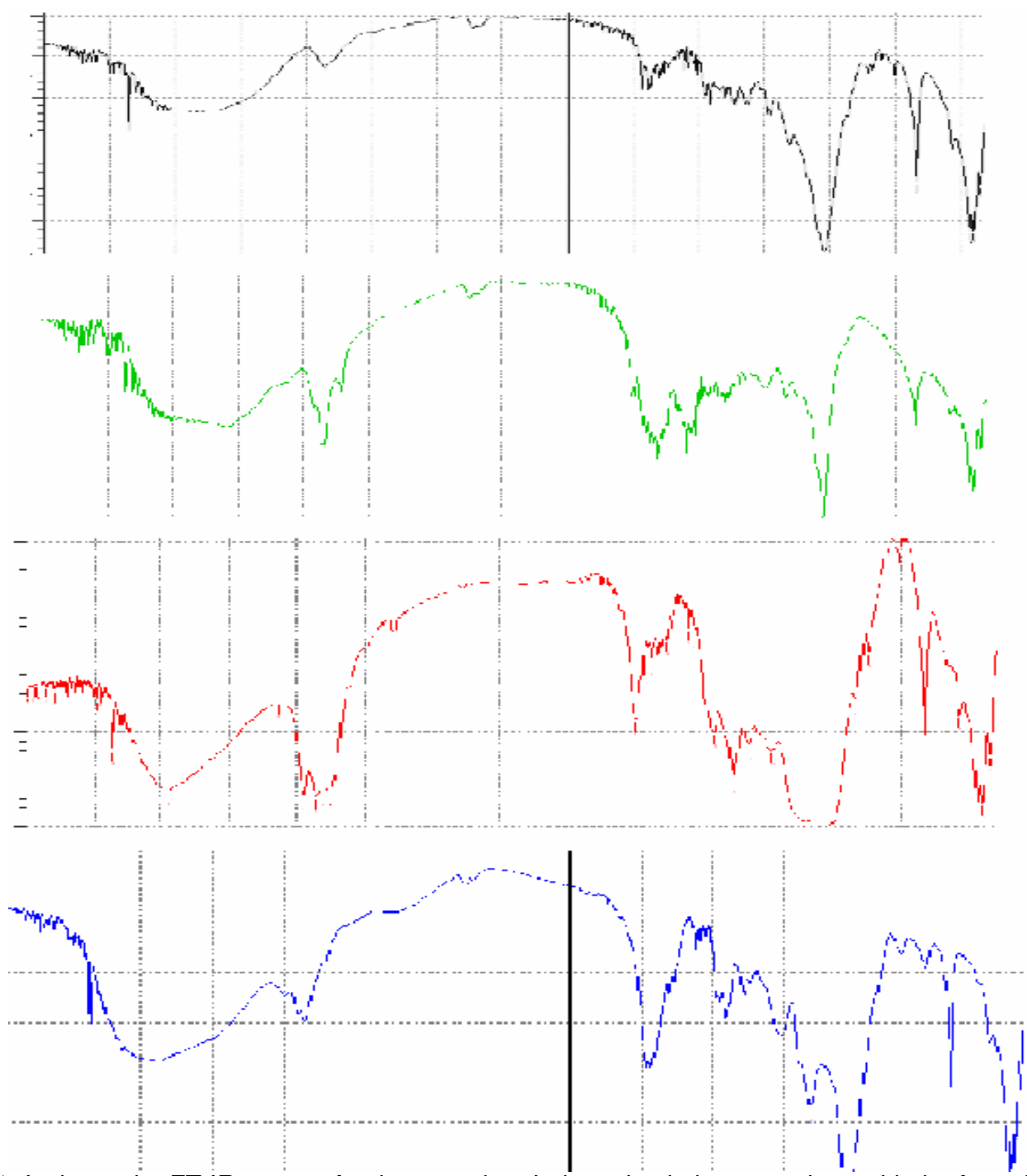

Fig. 1- It shows the FT-IR spectra for the pure drug Indomethacin in comparison with the formulations containing natural and synthetic polymers. (---) Indomethacin, (--) Egg albumin (---), Ethyl cellulose, (---) Eudragit. 


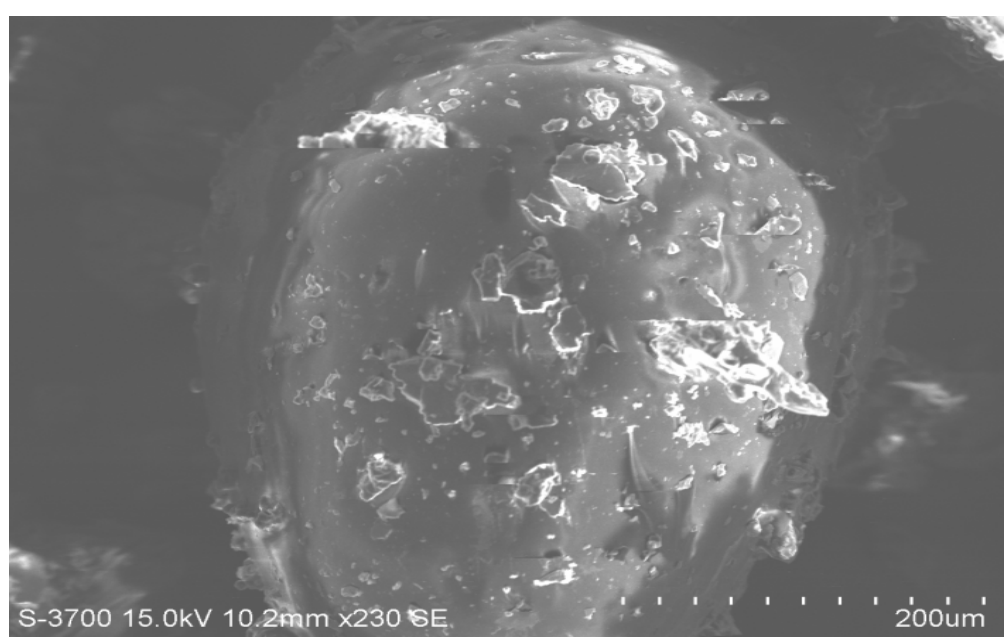

Fig. 2- Scanning electron microscopy of Egg albumin and Eudragit microspheres

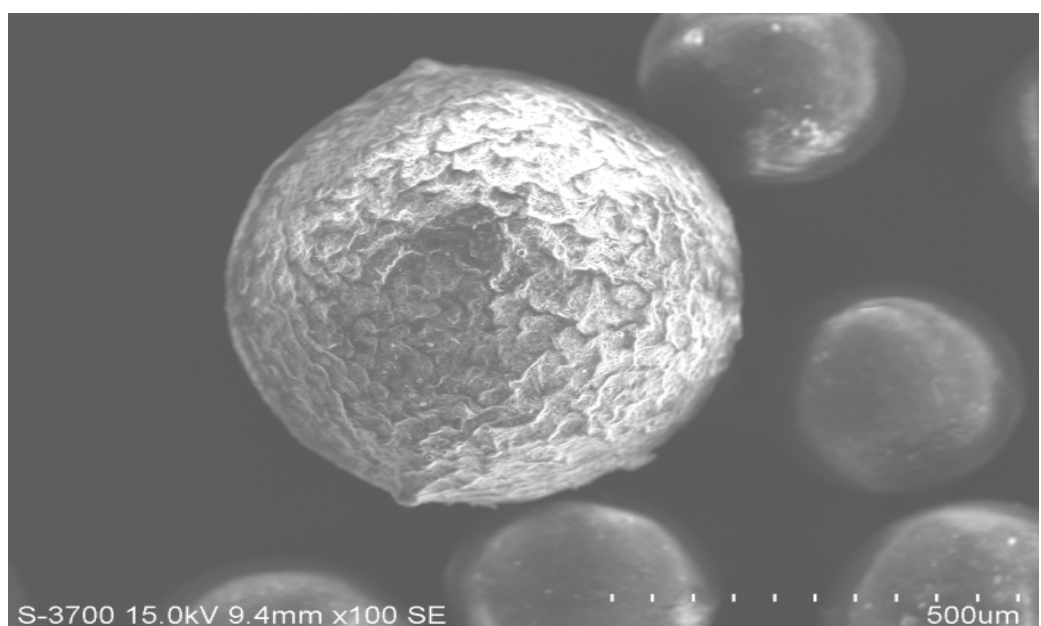

Fig. 3- Scanning electron microscopy of Egg albumin and Eudragit microspheres

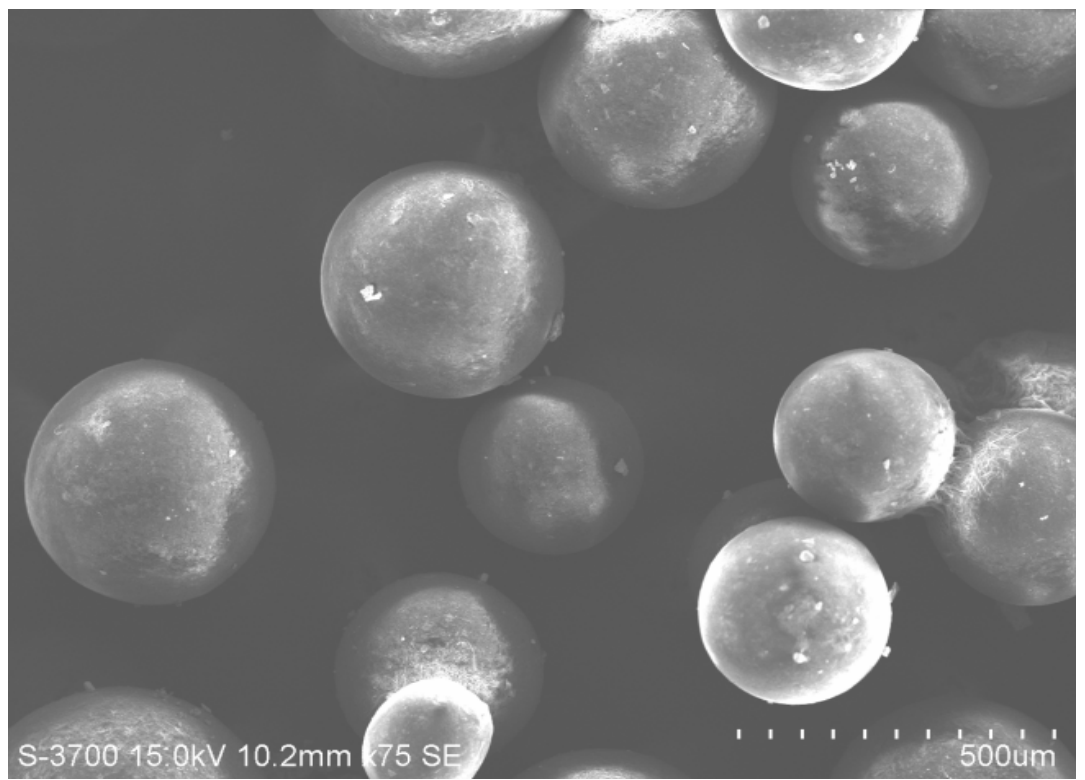

Fig. 4- Scanning electron microscopy of Ethyl cellulose microspheres 


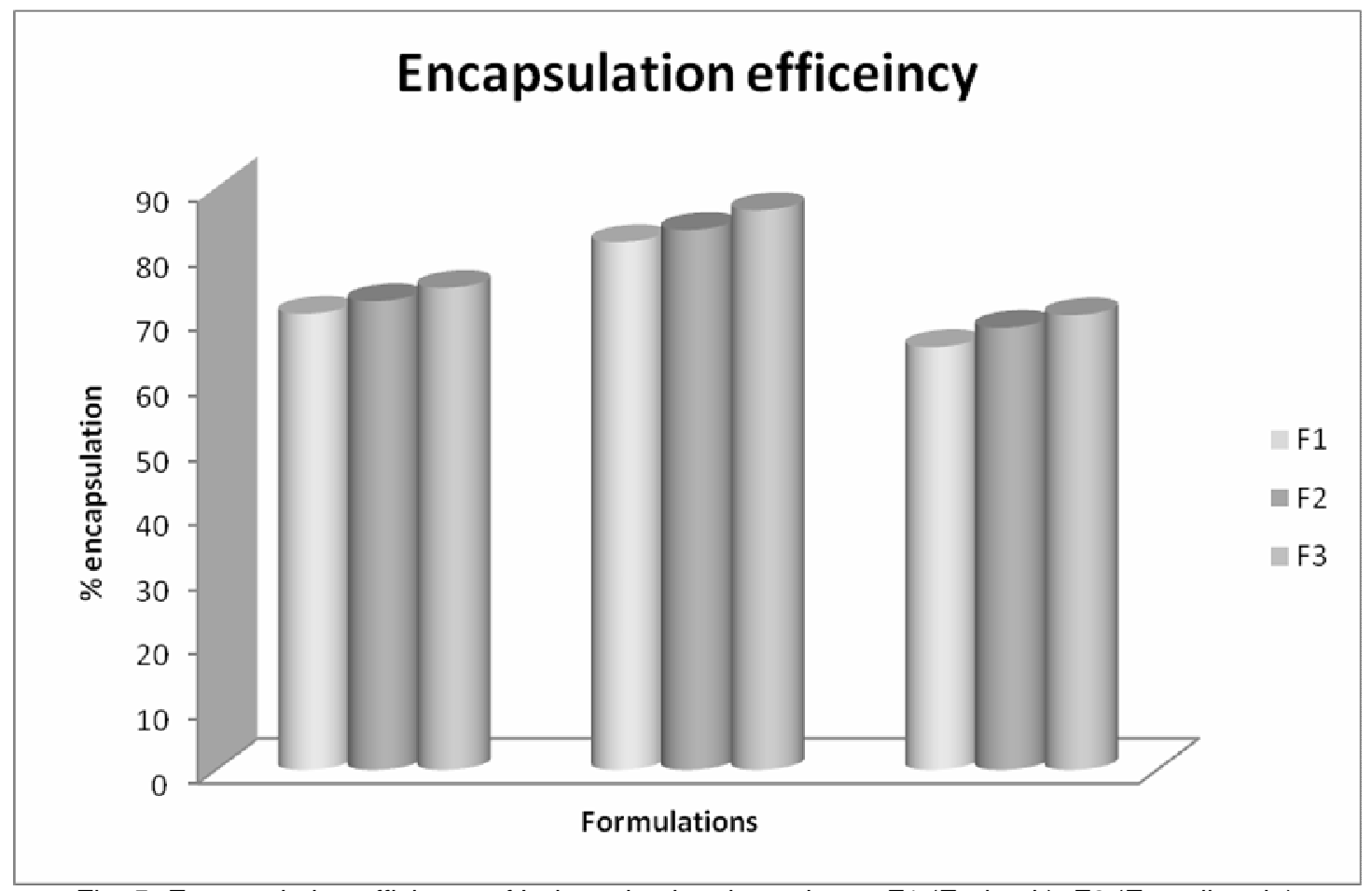

Fig. 5- Encapsulation efficiency of Indomethacin microspheres F1 (Eudragit), F2 (Egg albumin) and F3 (Ethyl cellulose)

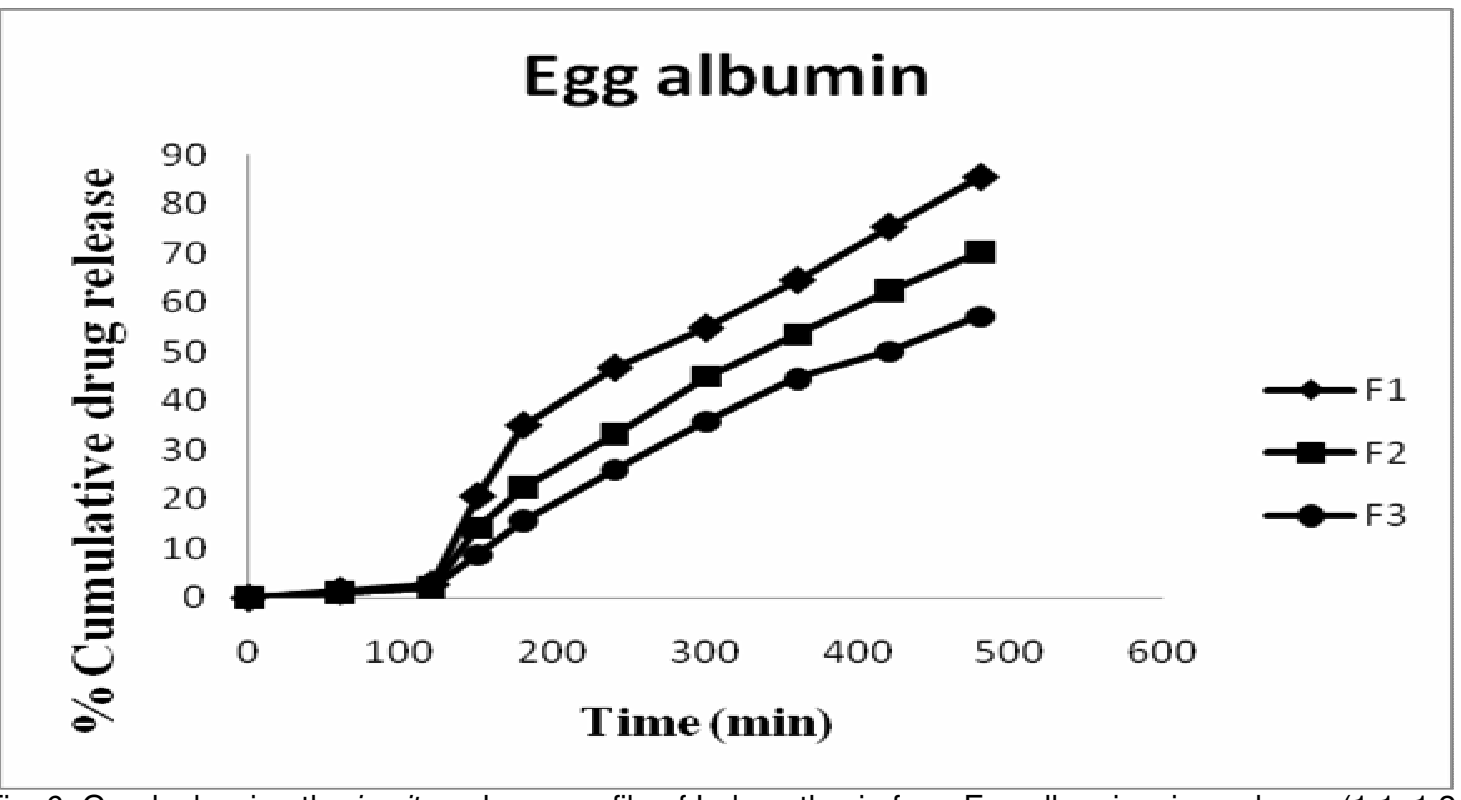

Fig. 6- Graph showing the in-vitro release profile of Indomethacin from Egg albumin microspheres (1:1, 1:2, $1: 3)$ 


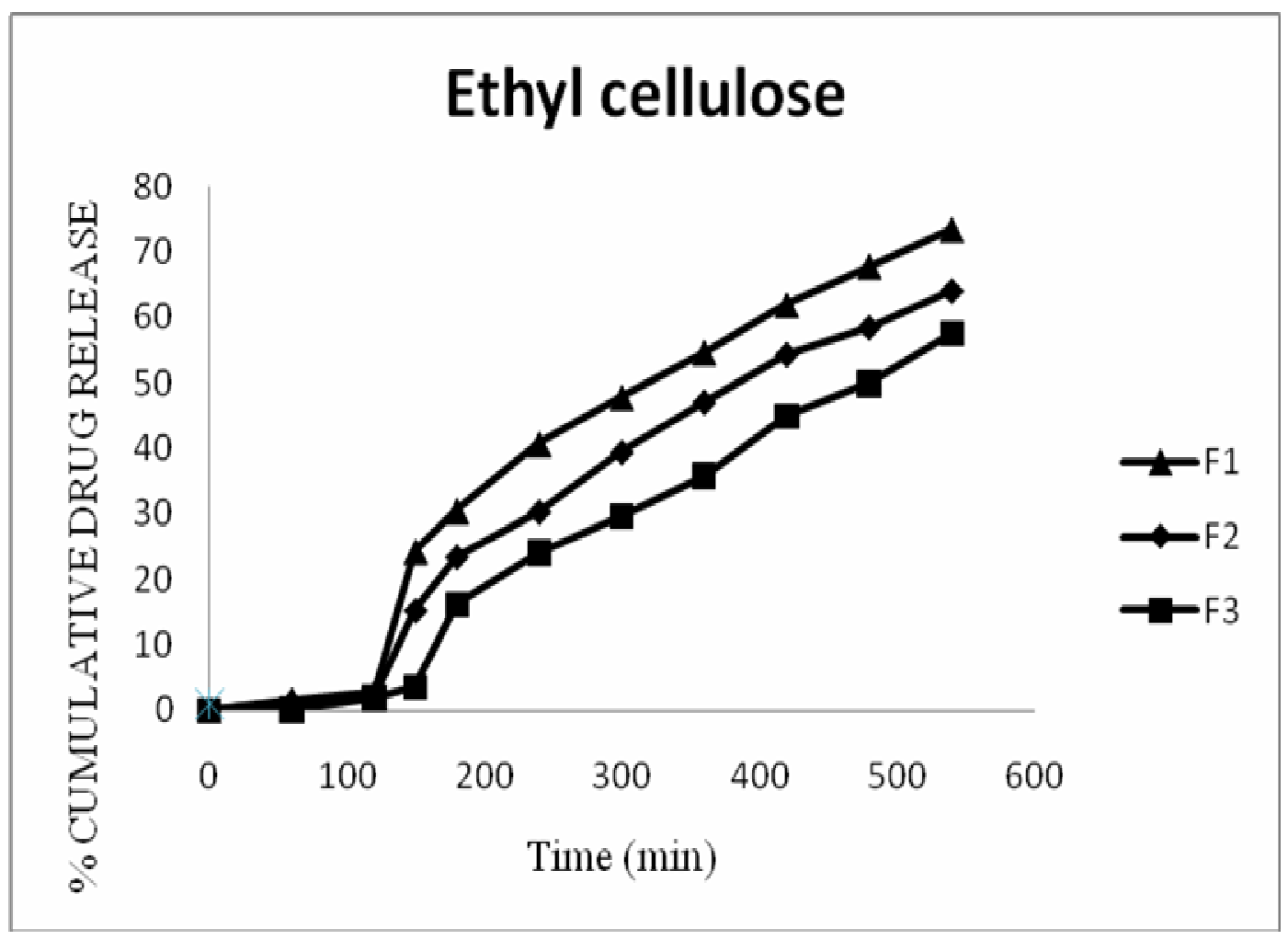

Fig. 7- Graph showing the in-vitro release profile of Indomethacin from Ethyl cellulose microspheres (1:1, $1: 2,1: 3)$

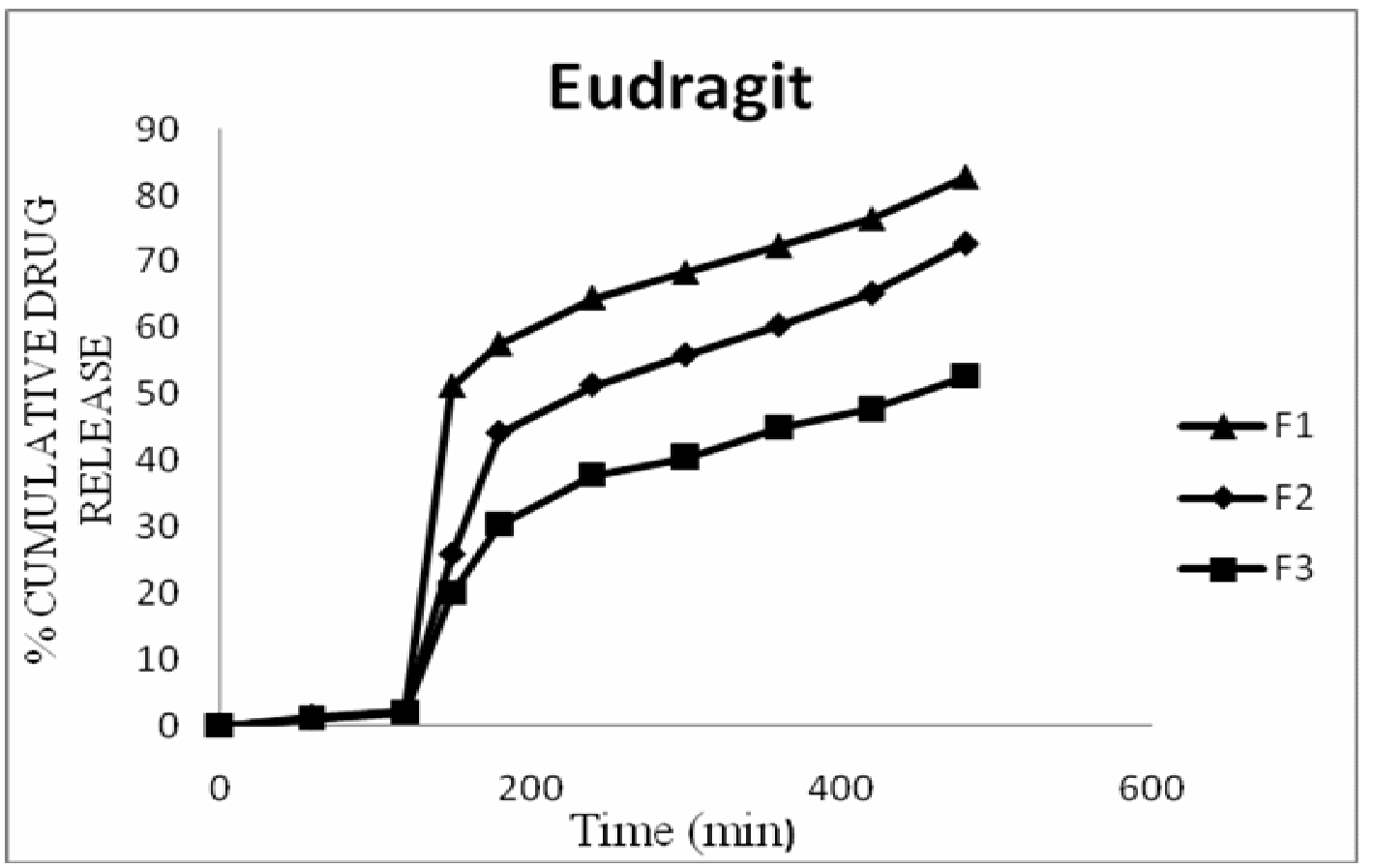

Fig. 8- Graph showing the in-vitro release profile of Indomethacin from Eudragit microspheres (1:1, 1:2 and $1: 3)$ 